Optimizing operational costs and service quality in dial-a-ride systems Peer-reviewed author version

MOLENBRUCH, Yves (2017) Optimizing operational costs and service quality in dial-a-ride systems. In: 4OR: A Quarterly Journal of Operations Research, 15(4), p. 435-436.

DOI: $10.1007 / \mathrm{s} 10288-017-0351-5$

Handle: http://hdl.handle.net/1942/25915 


\title{
Optimizing operational costs and service quality in dial-a-ride systems
}

\author{
Yves Molenbruch
}

August 11, 2017

This is a summary of the author's PhD thesis, supervised by An Caris and Kris Braekers and defended on May 24, 2017 at the Faculty of Business Economics of Hasselt University. The thesis is written in English and is available from the author upon request at yves.molenbruch@uhasselt.be. His research was funded by FWO (Research Foundation Flanders) and fits in the COMEX project (Combinatorial Optimization: Metaheuristics \& Exact Methods) of the Belgian Science Policy Office.

Dial-a-ride systems provide demand-dependent, collective transportation to people with reduced mobility. They have become indispensable in today's society, due to demographic evolutions and developments in the healthcare sector (e.g. the emergence of daycare centers for elderly). Providers of diala-ride services require vehicle routing algorithms to support their operational activities. This PhD thesis develops an algorithm that may assist providers in balancing the operational costs and quality level of their services, which allows them to implement a strategic quality policy on the operational level.

Due to the dynamic nature and the complexity of this routing problem, research to date has mainly focused on the development of metaheuristic solution algorithms. They usually minimize the total vehicle distance, while quality is ensured through minimum service level requirements. First, pickups or deliveries should respect a time window based on the users' preferences. Second, the users' ride times cannot exceed a predefined maximum duration. Imposing stricter quality requirements reduces the number of possibilities to combine multiple users in the same vehicle, which strongly influences the operational costs incurred by the provider. 
This traditional solution approach does not optimize the quality and forces providers to impose minimum service level requirements in advance. In contrast, this $\mathrm{PhD}$ thesis presents a metaheuristic routing algorithm which provides insight into the tradeoff of operational costs and service quality. It creates a Pareto set of routing solutions with respect to two objectives: the total vehicle distance (operational objective) and total user ride time (service-related objective). Experiments show that slight deviations from the minimal operational cost may considerably improve the service quality. Specific attention is devoted to the impact of real-life problem characteristics, such as heterogeneous user types and advanced routing properties. For example, the experiments are carried out on a real-life problem variant with combination constraints, meaning that certain users cannot be combined with other users or should be assigned to a driver with medical qualifications.

To assess the quality-related objective, the vehicles' time schedules should be computed such that the total ride time of the users is minimized. The only existing scheduling approach with this objective does not guarantee to find a feasible schedule for each feasible route and frequently produces deviations from the optimal schedule. This PhD thesis introduces a new heuristic scheduling procedure that strongly reduces the risks of such failures while requiring less computation time. Moreover, an extension of this procedure avoids that a user's excessive ride time may be converted into unnecessary waiting time.

Operational strategies applied by service providers may also influence the tradeoff of operational costs and service quality. If different providers operating in the same area share a similar quality vision, their operational costs can be reduced by means of joint route planning. This implies that user requests may be exchanged among providers, based on a centralized decision strategy that minimizes the joint costs incurred by the providers, while leaving the service quality unchanged. Experiments show that the resulting savings strongly depend on various operational characteristics (e.g. the number of requests, the location of the depots, the spatial clustering of demand, ...). In addition, such a centralized decision strategy can only hold in the long term if all providers agree on a gain sharing strategy to allocate these savings among each other.

Based on the aforementioned findings, this $\mathrm{PhD}$ thesis is concluded with a number of recommendations to the three stakeholders that may influence the balance of operational costs and service quality in dial-a-ride systems, being academic researchers, service providers and policy makers. Their decisions determine the extent to which the quality expectations of users, being the fourth main stakeholder, are satisfied. 